\title{
Biomarkers of Bladder Cancer in Urine: Evaluation of Diagnostic and Prognostic Significance of Current and Potential Markers
}

\author{
Daben Dawam \\ Medway NHS Foundation Trust \\ Medway Maritime Hospital \\ Associate Teaching Hospital, University of London, \\ United Kingdom
}

\section{Introduction}

The diagnosis of bladder cancer is generally made by cystoscopy and biopsy. Moreover, bladder cancer has a very high frequency of recurrence and therefore requires follow-up cystoscopy, along with urine cytology, as periodic surveillance to identify recurrence early. Cystoscopy is invasive and apt with complications like urine infection which sometimes lead to septicaemia with serious consequencies. Patient experience is most times not pleasant. Therefore, there needs to be a better way of surveillance for bladder cancer which is non-invasive and more acceptable to the patient experience. Consequently, urine biomarkers might be used to either supplement or supplant these tests.

Urinary bladder carcinoma, the fourth most common cancer in men and ninth most common in women results in significant morbidity and mortality.

Bladder cancer (urothelial carcinoma) typically presents as a tumour confined to the superficial mucosa of the bladder. The most common symptom of early bladder cancer is haematuria; however, urinary tract symptoms (i.e., urinary frequency, urgency and dysuria) may also occur. Most urologists follow the American Urological Association (AUA) guidelines for haematuria which recommend cystoscopic evaluation of all adults greater than 40 years old with microscopic haematuria and for those less than 40 years old with risk factors for developing bladder cancer. Confirmatory diagnosis of bladder cancer must by made by cystoscopic examination and biopsy which is considered to be the "gold standard."

At initial diagnosis, about 70 percent of patients have cancers confined to the epithelium or sub-epithelial connective tissue. Non-muscle invasive disease is usually treated with transurethral resection with or without intravesical therapy, depending on depth of invasion and tumour grade. However, there is a 75 percent incidence of recurrence in these patients with 10-15 percent progressing to muscle invasion over a five year period. Current follow-up protocols include flexible cystoscopy and urine cytology every three months for one to three years, every six months for an additional two to three years, and then annually, assuming no recurrence. 
While urine cytology is a specific test (from 90 percent-100 percent), its sensitivity is lower, ranging from 50 percent-60 percent overall and is considered even lower for low-grade tumours. Therefore, there has been interest in identifying tumour markers in voided urine that would provide a more sensitive and objective test for tumour recurrence.

\section{Background}

Bladder cancer is very common, ranking second only to prostate cancer for cancers of the urinary tract. Approximately 54000 new cases of bladder cancer are diagnosed and 12 000 people die from this disease every year in the United States alone. Most patients are diagnosed with superficial tumours, which can be completely resected. However, two-thirds of these patients will experience recurrence within 5 years, and almost $90 \%$ will have a recurrence by 15 years. Early diagnosis leads to better clinical outcomes, underscoring the importance of finding new ways for screening the general population. Currently, potential bladder tumour markers can be used in various clinical scenarios, including (14):

- Serial testing for earlier detection of recurrence;

- Complementary testing to urine cytology to improve the detection rate;

- Providing a less expensive and more objective alternative to the urine cytology test; and

- Directing the cytoscopic evaluation of patient follow-up.

The gold standard for the detection of urothelial neoplasia is cytologic examination of urothelial cells from voided urine, urinary bladder washings, and urinary tract brushing specimens in combination with cystoscopic examination 12,13. Because cystoscopy is an invasive procedure and urinary cytology suffers from low sensitivity and specificity, particularly for lower grade tumours, it is desirable to identify novel biomarkers for this cancer. Biochemical testing of urine is a non-invasive and less expensive procedure for diagnosing and monitoring this disease. Because none of the markers mentioned above has sufficient sensitivity and specificity, the quest for identifying additional bladder cancer biomarkers continues.

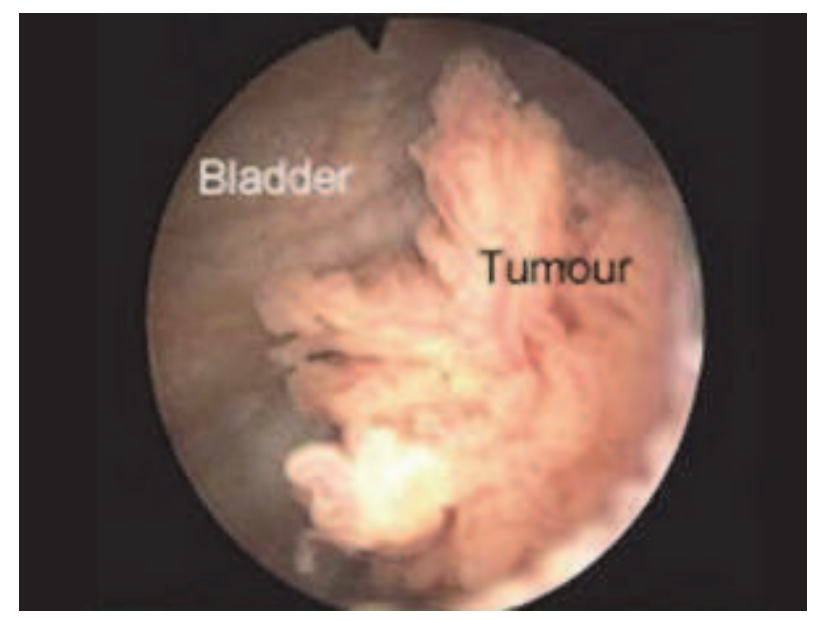

Fig. 1. Cystoscopic appearance of bladder tumour 

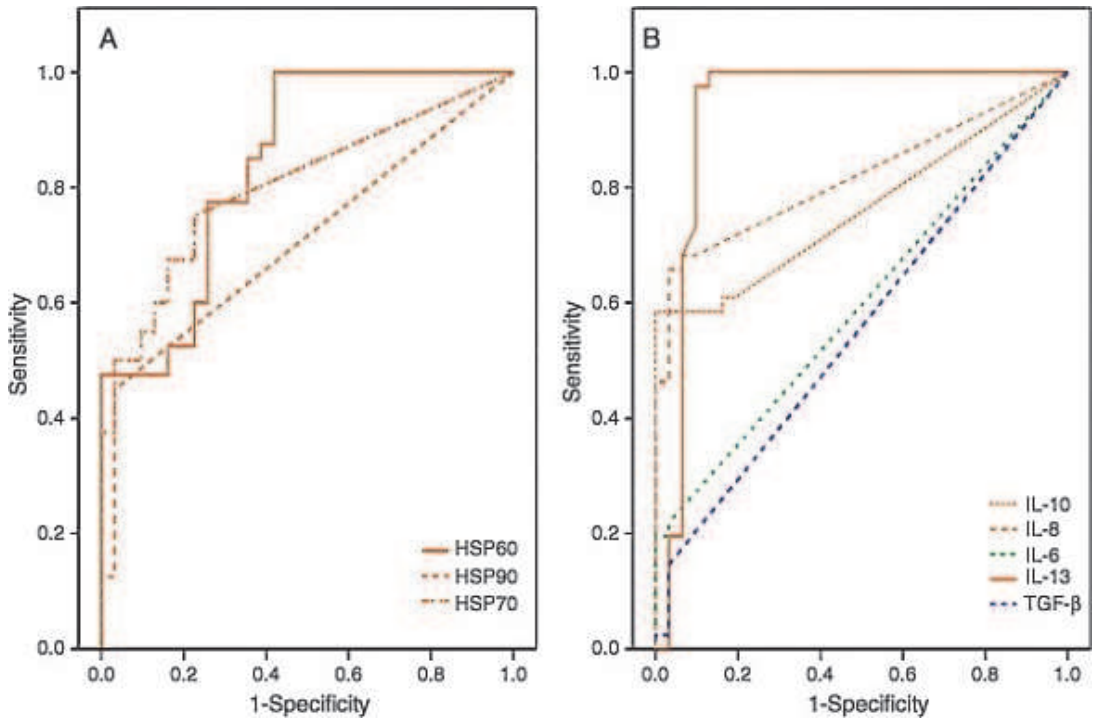

Fig. 2. Relationship between sensitivity and specificity

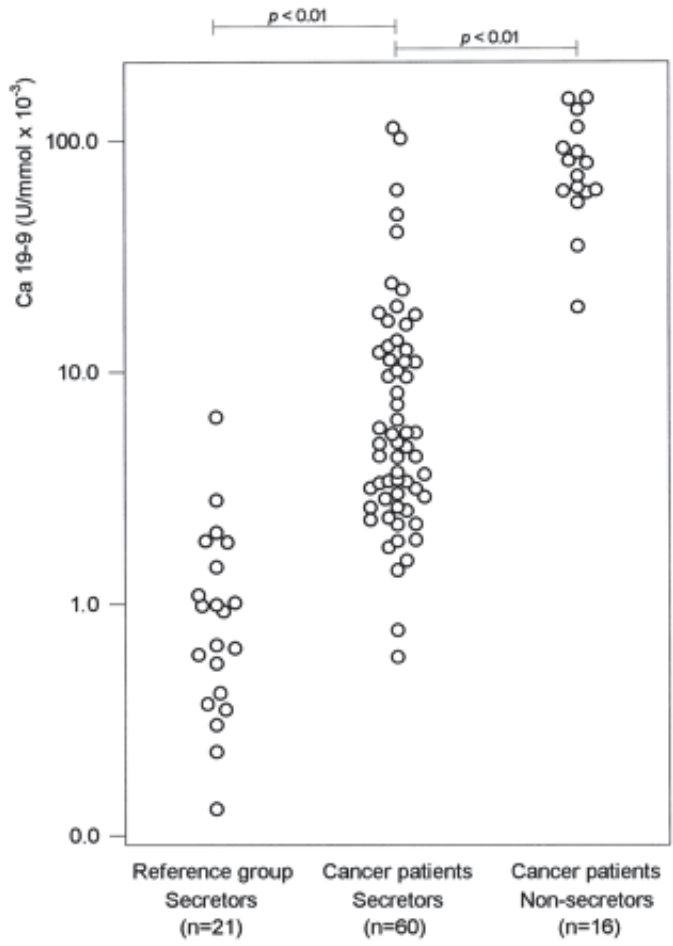

Fig. 3. Ca 19-9 levels in urine (Adapted from ClinChem.org) 


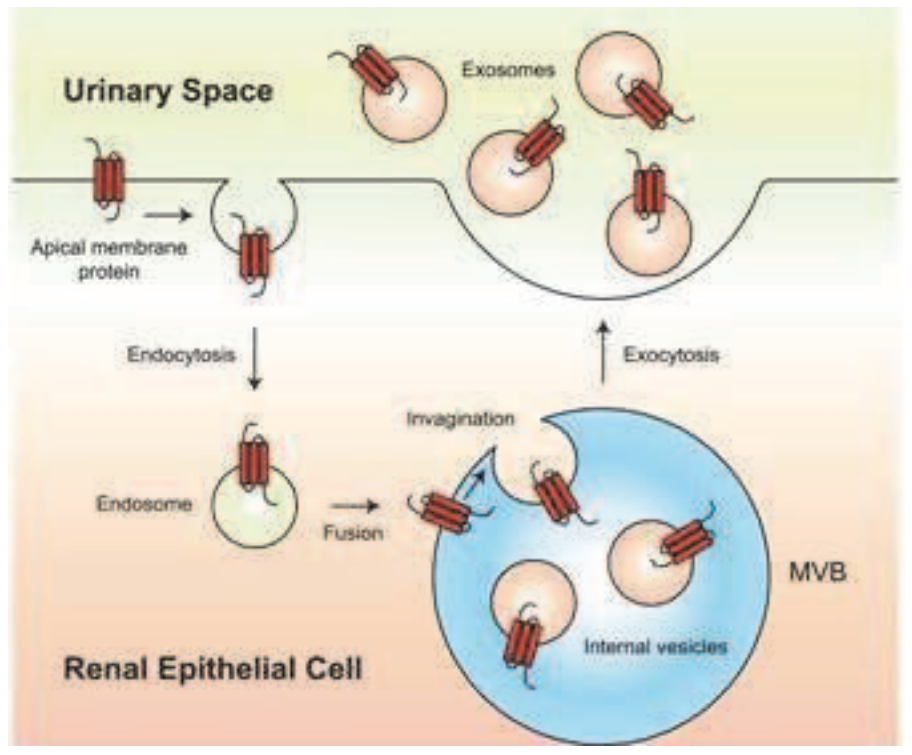

Fig. 4. Mechanism of cancer marker production and appearance in urine (Adapted from flipper.diff.org)

Kageyama et al. propose proteomic analysis of urine as a new way to identify bladder cancer biomarkers. Previously, Celis et al. utilised two-dimensional gel electrophoresis and developed a comprehensive database for bladder cancer profiles of both transitional and squamous cell carcinomas.

Biochemical testing of urine should be able to diagnose early bladder carcinoma because candidate informative molecules could be excreted into the urine during cancer development. Proteomic profiling of urine has been suggested as a diagnostic test for bladder carcinoma 11. In addition, many other biochemical molecules or genetic markers have been discovered that could be used to diagnose bladder carcinoma with fair sensitivity and specificity. Such molecules (or methods) include, but are not limited to, the following (the approximate diagnostic sensitivities and specificities are in parentheses): BTA stat (68\%; $66 \%)$; BTA-TRAK (71\%; 62\%); NMP22 (64\%; 71\%); telomerase (74\%; $89 \%)$; HA-HAase $(91 \%$; $86 \%)$; Immunocyt $(68 \% ; 79 \%) ; \mathrm{F} / \mathrm{FDP}(68 \% ; 86 \%) ;$ multicolor fluorescence in situ hybridization assays $(84 \%$; $90 \%)$; cytokeratins $(76 \% ; 84 \%)$; metalloproteinases $(60 \%$; $80 \%)$; and p53 mutation $(32 \% ; 100 \%)$. The most common noninvasive test, however, is voided urine cytology (VUC), which has a sensitivity of $\$ 50 \%$ and a specificity of $97 \% 12$. This test has higher sensitivity for higher grade tumors.

Through their studies, Kageyama et al. were able to identify a potential tumour marker, calreticulin, which is found in the urine of patients with bladder carcinoma. The authors used a differential display method of bladder cancer vs healthy urothelial tissue and mass spectrometry to identify proteins that are increased in cancer tissue. In addition to calreticulin, an endoplasmic reticulum chaperone, they found nine other candidate proteins that could constitute new biomarkers for bladder carcinoma. The authors confirmed their data with quantitative Western blot analysis, immunoprecipitation, and immunohistochemistry. Their reported sensitivity and specificity were $73 \%$ and $86 \%$, 
respectively, similar to the values reported for other biochemical bladder markers (see above). However, the diagnostic accuracy of their test was vulnerable to urinary tract infections.

The main question surrounding bladder cancer and urinary biomarkers is how these molecules can be used in clinical practice. Clearly, these tests are not useful for population screening because of their low sensitivity and specificity. In addition, none of the available tests is sufficiently accurate to replace cystoscopy in the investigation of a patient with a possible bladder tumour. VUC has relatively low sensitivity, especially for low-grade tumours, but it is currently the most specific test for bladder carcinoma. Consequently, when VUC is positive, it indicates a high-risk tumour that requires definitive treatment. VUC is currently used for monitoring of patients with known high-risk disease, and positive cytology with negative cystoscopy may indicate malignancy of the prostate or upper urinary tract.

Current guidelines suggest that low-risk patients should be surveyed once a year with cystoscopy and high-risk patients at 3-month intervals. Currently, cystoscopy is always combined with VUC. Because, as mentioned earlier, new urinary bladder tests such as BTA or NMP22 could detect lower-grade disease recurrence with higher sensitivity than VUC, it could be worthwhile to consider including one or more of these tests in the routine followup of patients with bladder carcinoma. However, large prospective studies will be necessary to test the clinical utility of these assays against cytology. Such trials could show the value of these new tests in reducing the frequency of cystoscopy and in contributing to the earlier and more sensitive detection of disease recurrence, leading to earlier therapeutic interventions and, fortunately, to improved clinical outcomes.

In conclusion, bladder cancer biomarkers have proliferated more than any other class of cancer markers over the last 10 years. We now have at hand a multitude of molecules that can be measured with automated, inexpensive, quantitative assays in urine. These markers may aid in the monitoring of patients with bladder carcinoma and have the potential to reduce the number of follow-up cystoscopy, thus reducing healthcare costs and patient discomfort and, at the same time, detecting relapsing disease more effectively than VUC. It is time to test these new possibilities with prospective clinical trials.

\section{Evaluation of individual markers}

Urine-based marker tests are being developed to fill some of the remaining needs. These newer tests are more accurate in detecting low-grade bladder cancer, so they are especially useful in monitoring for recurrence, may significantly improve and simplify workup, diagnosis, and follow-up, and hopefully allow for detection of disease at an earlier stage, thus improving the chances of curative therapy.

The urine marker assays discussed here have shown enhanced sensitivity in detecting bladder cancers. However, each still requires further validation and testing in clinical trials to determine how best to apply these tools for in individual patients. In recent years several of the newer tests are being used by urologists as another weapon in the arsenal. Although immunological markers are superior to standard urine cytology, at the present time urine bound tests are not specific enough to completely replace cystoscopy as a definite diagnostic tool.

In order to understand what these tests are about it's helpful to have an understanding of Sensitivity vs. Specificity: 
A diagnostic test is one that predicts the presence of a disease. An ideal diagnostic test would always give the right answer, with a positive result in everyone with the disease and a negative result in everyone else - and would be quick, safe, simple, painless, reliable, and inexpensive, as well. Since no current diagnostic test is ideal, we need to evaluate each of them for their clinical usefulness. In practice, for any diagnostic test there is a trade-off between sensitivity and specificity. In cancer diagnosis, the need for this trade-off is rooted in the fact that cancer arises from our own tissues. It is not completely "foreign" to our systems like a virus or bacterium is.

It's important to remember that there are four possible results when a diagnostic test is run: True positive - when the test is positive and the patient does have the disease False positive - when the test is positive but the patient does not have the disease True negative - when the test is negative and the patient does not have the disease False negative - when the test is negative but the patient does have the disease Here's another way of looking at this (often referred to as a "truth table"):

\begin{tabular}{|l|l|l|}
\hline $\begin{array}{l}\text { Test } \\
\text { Result }\end{array}$ & $\begin{array}{l}\text { The disease being tested for is } \\
\text { present }\end{array}$ & $\begin{array}{l}\text { The disease being tested for is not } \\
\text { present }\end{array}$ \\
\hline "Positive" & True positive & False positive \\
\hline "Negative" & False negative & True negative \\
\hline
\end{tabular}

Calculating the disease sensitivity and specificity are ways of evaluating diagnostic tests, using the four possible results.

Sensitivity - is the ability of a test to correctly identify a positive specimen, and it tells you how good the test is at identifying the disease. Statistically, it's the proportion of patients with the disease who have a positive result, that is, the number of "true positives" out of all the situations where the disease is present.

For example, 100 patients with cancer are tested using a test that detects tumours. There are 80 positive results and 20 negative results. This means the test has a sensitivity of $80 \%$ - it correctly identified 80 of the 100 cancers - and it gave 20 false negative results.

Specificity - is the ability of a test to correctly identify a negative specimen, and it tells you how good the test is at identifying when the disease is absent. The statistical way of looking at this is the proportion of patients without the disease who have a negative test, that is, the number of "true negatives" out of all the situations where the disease is not present.

For example, 100 normal, healthy individuals are tested using a test that detects tumours. There are 80 positives and 20 negatives. This means the test has a specificity of $20 \%$ - it correctly identified 20 of the 100 negative specimens - and it gave 80 false positive results.

Both sensitivity and specificity are very important, and they can both be influenced by various factors, such as the characteristics of the population tested or the value used as a cut-off for the test (above which the test is positive and below which it is negative). A test with low sensitivity and many false negative results will fail to detect the tumour in a large portion of the patients being tested, while a test with low specificity with many false positive results may lead to unnecessary invasive or expensive procedures and cause undue alarm.

Many, but not all, patients report they would rather be "scared for nothing" than miss a tumour, and are therefore most interested in tests with high sensitivity.1 


\section{BTA stat test and the BTA TRAK assay}

The original Bard BTA Test, which continues to be referred to in the literature from time to time, was a latex agglutination test detecting bladder tumour-associated antigen and is no longer distributed in the US. It is important to note that it has been replaced by two newer tests based on significantly improved technology with much better sensitivity and specificity.

Both of the new tests detect a human complement factor H-related protein (hCFHrp) which has been shown to be produced by several human bladder cancer cell lines, and by human bladder cancers, but not by other epithelial cell lines (Kinders, Clin Cancer Res 4:2511, 1998). It is thought that factor $\mathrm{H}$ acts to protect the tumor cell from the body's natural immune system (Corey, J Biol Chem 275:12917, 2000). Both the BTA stat and BTA TRAK tests can provide valuable but slightly different information for the bladder cancer patient and her doctor.

The BTA stat Test is a qualitative (positive or negative) test provided in a disposable format similar to a home pregnancy test. It uses five drops of urine and can be read in five minutes by the appearance of a coloured line in the patient window, while a coloured line appears in a "check" window to indicate the test is working properly. This test is cleared in the US for use by clinical laboratories, the physician or his staff right in the office, or even by the bladder cancer patient at home (with a physician's prescription). To date, it is the only tumour marker in the United States with this status. Besides being highly sensitive, fast, and easy to use, with a unique availability to be run by the physician and/or the patient, this test is significantly less costly than other diagnostic tests or cytology.

The BTA TRAK Assay is a quantitative immunoassay test and provides a numerical result of the hCFHrp level. Like the NMP22 test, urine must be sent to a reference laboratory where the test is performed by professional technologists. In addition to knowledge of the specific level, an advantage of the BTA TRAK test is the ability to monitor the rise or fall of hCFHrp.

Numerous clinical studies have been conducted with the new BTA tests. Most reports state findings in terms of "sensitivity" and "specificity." Briefly, sensitivity is the ability of the test to correctly identify a positive specimen, and specificity is the ability of the test to correctly identify a negative specimen.

\subsection{BTA stat test studies}

In the most recent study (June 2000) and the largest of its kind to date, Raitanen reported the overall sensitivity of BTA stat as $82 \%$, and cytology as $30 \%$. In another study, Pode reported $100 \%$ BTA stat sensitivity in tumors of stage T2 or higher, grade III, and all tumors greater than 2cm (Pode, J Urol 161:443, 1999). Specificity of the BTA stat Test has been reported as 72-95\% (Sarosdy, Urology 50:349, 1997) and 98\% in healthy individuals (Raitanen, Scand J Urol Nephrol 33:234, 1999).

\subsection{BTA TRAK assay studies}

In one study, the overall sensitivity of the BTA TRAK Assay was reported as $72 \%$ with a specificity of $75-97 \%$ (Ellis, Urology 50:882, 1997). Heicappell again reported an overall sensitivity of $72 \%$, with $97 \%$ specificity in healthy individuals. He also reported that BTA 
TRAK levels reflect tumour stage and grade, with levels in superficial bladder cancer at high risk of tumour progression significantly higher compared to low and intermediate grade superficial cancers (Heicappell, Eur Urol 35:81, 1999).

\subsection{Comparison studies}

In a study conducted at the Mayo Clinic, several urine tumour markers were evaluated, including urine cytology, BTA stat, NMP22, fibrin/fibrinogen degradation products (FDP), telomerase, chemiluminescent hemoglobin and hemoglobin dipstick. The telomerase test presented the highest combination of sensitivity and specificity for screening. However, other researchers have had difficulty reproducing the telomerase results of this study, possibly due to the technical difficulties of running the test. It's also important to note that telomerase is a "Research Use Only" test, and has not received FDA clearance for marketing in the US. In the same study, the BTA stat Test was shown to have the best overall sensitivity (74\%), and the best sensitivity for T1-T3 and primary tumour detection (Ramakumar, J Urol 161:388, 1999).

Another comparison study (Giannopoulos, Urology 55:871, 2000) showed that the BTA stat Test was more sensitive than cytology in all stages and grades except G3, while NMP22 was more sensitive than cytology only in stage Ta and Grade 1 and 2. The BTA stat Test also had higher sensitivity than NMP22 in all stages and grades.

It is also important to note that in both of the BTA tests, and with NMP22 as well, results can be compromised if there is a urinary tract infection, inflammation, or kidney stones present, if there has been recent trauma to the bladder, or if the specimen is collected by catheter. The paper by Sharma, for example, shows the dramatic increase in specificity when these conditions are excluded from testing (Sharma, J Urol 162:53, 1999). As with any test, for the results to be most useful they should be interpreted in light of all the medical and clinical information available.

\section{NMP22 'Bladder check'}

In a study comparing cystoscopy, cytology, and Bladder Check; the NMP22/Bladderchek test had a considerably higher detection rate than cytology (67\% vs. 20\%). Cystoscopy detected $86 \%$ of bladder cancers.

More cost effective than cytology, the Bladder check test could also be a good adjunct to cystoscopy. The test costs in the range of $\$ 20$ to $\$ 25$, which Medicare reimburses for both bladder cancer monitoring and detection. It is a waived test under the Clinical Laboratory Improvement Amendments (CLIA).

While the test showed a high negative predictive value, it produced a false-positive result in 19 of the 194 patients without bladder cancer. Dr. Tomera advised that such patients need to be watched closely. Earlier data by Mark Soloway, MD, has shown that bladder cancer will be found in $70 \%$ of these individuals during the following 3 to 6 months (J Urol 1996; 156:363-7).

NMP22's core technology is based on the level of nuclear matrix proteins (NMPs) that are detected in body fluids. These levels are correlated to the presence of early-stage cancerous abnormalities, which have been validated in multiple clinical studies. The technology was discovered at the Massachusetts Institute of Technology and licensed to Matritech. 


\section{FISH}

Florescence in situ hybridization (FISH) is an assay which uses a mixture of fluorescent labeled probes to assess urinary cells for chromosomal abnormalities associated with malignancy.

In a study at the Mayo clinic, researchers found that urine cytology detected cancerous cells in only 57 percent of the patients with bladder cancer while the FISH test picked up more than 95 percent of the high grade cancers, which are the most dangerous and important group of bladder cancers because they have a high probability of progressing to potentially incurable muscle-invasive bladder cancer. Cancers the test missed were low-grade tumours, which are less dangerous and have only a 3 to 5 percent chance of progressing to a higher stage tumour over five years. The FISH test also detected recurrence of the cancer three to six months earlier than by the cytology. This earlier detection capability should allow treatment to be initiated earlier and possibly give the patient a greater chance for survival, he said.

Fluorescence-in-situ-hybridization (FISH) for multiple centromeric probes has previously been shown to be a very sensitive test for diagnosing UC, however the test was limited by the requirement of multiple cytospins to evaluate 4 or more probe sets. Recently a new commercial test (VYSIS) for evaluating urinary cytology became available in which 4 probes are simultaneously evaluated on a per cell basis on a single cytospin. We performed a pilot study to test the efficacy of the new FISH test compared to standard urine cytology. This study showed that the multi-colour FISH probe test was more sensitive than cytology, easily performed and yielded a high number of cells with numerical chromosomal aberrations.

\section{DiagnoCure's ImmunoCyt ${ }^{\mathrm{TM}}$ bladder cancer monitoring test}

ImmunoCyt $\mathrm{t}^{\mathrm{TM}}$ is a $510(\mathrm{k})$ cleared, by the FDA, qualitative direct immuno-cytofluorescence assay, intended for use in conjunction with cytology to increase the overall sensitivity for the detection of tumor cells exfoliated in the urine of patients previously diagnosed with bladder cancer.

ImmunoCyt $\mathrm{t}^{\mathrm{TM}}$ contains a cocktail of three monoclonal antibodies labeled with fluorescent markers. The cocktail of antibodies have been shown to react with a mucin glycoprotein as well as to be specific to a glycoform of CEA. The test detects cellular markers specific for bladder cancer in exfoliated cells isolated from urine sample. This non-invasive test, when coupled with urine cytology proves to be more sensitive than urine cytology alone or other currently available tumour markers.

The current standard method for non-invasive detection of bladder cancer is urinary cytology, which consists of identifying the presence of cancer cells in urine. Urinary cytology has high specificity but poor sensitivity, typically no greater than $30 \%$ to $45 \%$. This sensitivity varies according to the stage and grade of the tumor.

ImmunoCyt $\mathrm{T}^{\mathrm{TM}}$ is carried out in parallel with cytology to improve cytology's sensitivity at detecting tumour cells in the urine of patients, especially those with low stage, low grade tumors. The concomitant use of classical cytology and ImmunoCytTM can substantially improve the detection of bladder cancer. As shown in the ImmunoCytTM performance analysis (cumulative data from eleven publications and presentations from 3,203 cases), a sensitivity of $88 \%$ has been obtained when both cytology and ImmunoCyt ${ }^{\mathrm{TM}}$ were used together. 
A multi-centre study in the United States, published in the Journal of Urology, concluded: ImmunoCyt ${ }^{\mathrm{TM}}$ enhances the sensitivity of cytology, which is a specific but not a sensitive method for detecting bladder cancer. The ability of this immuno-cytochemical test to detect low grade, superficial, small tumours makes it the most suitable available marker to test for monitoring strategies in patients with low risk bladder cancer. Performance of urine test in patients monitored for recurrence of bladder cancer: a multi-centre study in the United States.

\section{FDP-Fibrin/Fibrinogen Degradation Products}

FDP has shown high sensitivity even for low-grade and non-invasive tumours, and its diagnostic ability could be superior to NMP22 according to a recent study

The FDP test detects the presence of fibrin and fibrinogen degradation products in urine. It is a simple test that can be performed in the office, and results are available in about 10 minutes. Fibrin and fibrinogen degradation products are protein fragments generated by the action of the fibrinolytic system on fibrin and fibrinogen. Plasma proteins leak from blood vessels in tumours into the surrounding tissue. Clotting factors rapidly convert the fibrinogen in the plasma into an extravascular fibrin clot, which is degraded by plasmin and activated by urokinase. The FDP test can detect these degradation products and is positive in two thirds of patients with bladder cancer. The FDP assay is more accurate than urine cytology and has high specificity (negative in $96 \%$ of healthy subjects). The FDP test was found to be superior to the BTA test in at least one study*.

Telomerase is another substance currently being assessed for its potential usefulness in diagnosing transitional cell cancer (TCC) and in monitoring for recurrence. It will soon be made available to doctors and patients. Telomerase is a ribonucleoprotein enzyme responsible for production of telomeres, which are DNA sequences that occupy the ends of chromosomes and protect their integrity during DNA replication and may be involved in the immortalization of a cancer cell ${ }^{3}$

\section{Comparison of screening methods in the detection of bladder cancer}

In a study done in '99, researchers prospectively evaluated and compared the sensitivity and specificity of urine cytology, BTA stat, NMP22, fibrin/fibrinogen degradation products (FDP), telomerase, chemiluminescent hemoglobin and hemoglobin dipstick to detect bladder cancer ; within each tumour grade and stage telomerase had the strongest association with bladder cancer among all tests (69\% overall concordance). Telomerase was positive in $91 \%$ of the patients (10 of 11) with carcinoma in situ. The combination of sensitivity and specificity (70 and $99 \%$, respectively) was the highest for bladder cancer screening in these patients. Telomerase outperformed cytology, BTA stat, NMP22, FDP, chemiluminescent hemoglobin and hemoglobin dipstick in the prediction of bladder cancer. 4

Telomerase - According to a study published in JAMA (2005; 294:2052-6) Italian researchers reported the assay showed $90 \%$ sensitivity and $88 \%$ specificity. Specificity increased to $94 \%$ for those aged 75 years or younger. The same predictive capacity of activity levels was observed for patients with low-grade tumours or with negative cytology results. In particular, sensitivity was $93 \%, 87 \%$, and $89 \%$ for tumour grades 1,2 , and 3 , respectively. 
Although the test is proven to identify low-grade tumours, it is not recommended for use in routine screening programs because of the low incidence of bladder cancer and should be aimed at high-risk subgroups, noted the authors, from Morgagni-Pierantoni Hospital, Forli. Theoretically, urine telomerase appears more promising than do non-invasive tests for bladder cancer to date. The main advantages of the test, are that it is non-invasive, can be performed under local anaesthetic, and is significantly less expensive, at $\$ 20$, than the approximately $\$ 100$ for cystoscopy or $\$ 50$ for urinary cytology. It could be a good marker for high-risk screening groups. Furthermore, it shows a high sensitivity for the diagnosis of low-grade tumours that can escape detection during cytological examination. Results are usually available in 2 to 3 days.

\section{Hyaluronidase and hyaluronic acid}

Hyaluronidase seems to be directly involved in tumour growth and progression, and recent reports have shown this marker has high accuracy in detecting bladder cancer and evaluating its grade, Hyaluronidase and hyaluronic acid are associated with induction of angiogenesis. It has been shown that Hyaluronic acid (HA), the urinary HAase levels of intermediate (G2) to high- grade (G3) bladder cancer patients are five- to seven-fold elevated as compared to those of normal individuals and patients with other genitourinary conditions or low-grade (G1) bladder cancer. The increase in urinary HAase levels is due to the secretion of a tumour-derived HAase which is elevated eight-fold in G2/G3 tumour tissues. The HAase in bladder tumour tissues is secreted by tumour epithelial cells and is associated with the invasive/ metastatic potential of the tumour cells. ${ }^{5}$

Researchers from Brazil investigated the usefulness of HA for the detection of residual tumours that may remain after incomplete TUR. ${ }^{10}$ The authors concluded that HA- in addition to being one of the best markers for the initial evaluation of bladder carcinoma- can be used to determine the presence of a residual tumour. This is associated with poor prognosis. Furthermore, haematuria does not seem to influence the content of urinary HA. Other tumor markers such as FISH (Fluorescence in Situ Hybridization) and NMP22 might be affected by instrumentation and therefore could not be evaluated this early.

\section{Low values of urinary HA after TUR indicate a favourable prognosis and could probably avoid the second procedure}

The researchers suggest that after more experience and follow-up using this assay in the clinical setting, it might be possible to predict not only the cases with residual tumour, but also those who require early radical surgery or those in whom this can be delayed.

In addition to being a good marker in the initial evaluation of bladder carcinoma thanks to its excellent sensitivity (83.1\%) and specificity (90.1\%), HA potential uses include follow-up, prognostic evaluation, preventing unnecessary interventions and/or to indicate cases where early radical intervention is necessary.10

\section{BLCA-4}

Robert H. Getzenberg and colleagues at the University of Pittsburgh, USA have identified several components of the nuclear matrix, one of which is called BLCA-4, that differentiate human bladder tumour cells from normal bladder cells. Normal samples from unaffected 
individuals did not react with the antibody, and importantly, BLCA-4 appears to be present throughout the bladder (i.e., in both normal and tumour areas) in bladder cancer patients. This "field effect" permitted development of a urine immunoassay for BLCA-4 that detects the presence of tumour anywhere in the bladder, regardless of stage or grade. The BLCA-4urine immunoassay has a specificity of $100 \%$ and a sensitivity of $95 \%$. According to Dr. Getzenberg, the assay is currently being tested by the Pittsburgh researchers in a clinical trial of individuals at high risk for bladder cancer. 6

Using a prospectively determined cut-off, 67 of the 75 samples from patients with bladder cancer were positive for BLCA-4, resulting in an assay sensitivity of $89 \%$. Also, 62 of the 65 samples from individuals without bladder cancer were negative for BLCA-4, resulting in an assay specificity of $95 \%$. The authors concluded that the high sensitivity and specificity of the sandwich BLCA-4 immunoassay may allow for earlier detection and treatment of disease, thus greatly improving patient care. 7

BLCA-4, appears to be associated with a "field effect" of the disease, and in clinical trials is able to separate individuals with bladder cancer from those without the disease with high sensitivity and specificity. BLCA-4 is a bladder cancer marker that is highly specific and occurs early in the development of the disease. It appears to be a transcription factor that may play a role in the regulation of the gene expression in bladder cancer. BLCA-4 is a marker with significant clinical utility that may have an active role in the disease.

\section{Other proposed markers}

DD 23 monoclonal antibody recognizes a $185 \mathrm{kDa}$ antigen expressed by bladder cancer cells and has been proposed as an adjunct to cytology for the detection of bladder cancer. Urine fibronectin and chorionic gonadotropin (protein and mRNA transcript) may also be markers for transitional cell carcinoma of the bladder .

\section{Role of urine markers in early detection of bladder cancer}

Almost all cases of bladder cancer are found during the work-up of patients who present with haematuria (71), but most cases of haematuria are not caused by bladder cancer. Urologic disease is detected in $10 \%$ of subjects who present with haematuria, and bladder cancer is detected in fewer than half of these subjects $(72,73,74)$. The work-up of patients with haematuria is costly and often requires cytology, cystoscopy, intravenous urography or computed tomography (75). Thus, tumor markers could be useful in identifying the patients in this high-risk group, which requires more intensive clinical work-up for bladder cancer. Zippe et al reported on the value of the urine NMP22 test in the evaluation of 330 patients with haematuria (76). The NMP22 test when used with a cut-off value of $10.0 \mathrm{u} / \mathrm{ml}$ detected all 18 cases of bladder cancer with 45 false positive cases (sensitivity, 100\%; specificity, $85 \%$ ). In this study, 267 unnecessary cystoscopy could have been avoided if cystoscopy had been directed by the NMP22 test. In a clinical trial submitted to the Food and Drug Administration (as Pre-Market Approval Data), the NMP22 test was elevated in $69.6 \%$ of 56 bladder cancer that were detected in the high risk group. In this report, the specificity was $67.7 \%$ (77). The NMP22 test has been cleared by the FDA for use as an aid to diagnose bladder cancer in individuals with risk factors or who have symptoms of bladder cancer. It is highly likely that other urine markers (e.g. BTA, UroVysion and Immunocyt) may also have value for cancer detection in subjects who present with haematuria. The high false 
positive rate is the major criticism of the urine-based tests when they are used to assess patients who present with haematuria or are used in patient surveillance. The low false negative rate of these tests is their strength, leading to a high negative predictive value that effectively rules out disease in a significant proportion of patients, thereby eliminating unnecessary clinical work-ups for bladder cancer.

\section{Role of tissue markers for prognosis}

Considerable research effort continues to be directed towards the identification of markers that predict the aggressive potential of superficial bladder tumors. Such information could lead to more effective surveillance protocols and permit more aggressive treatment of those patients with tumors most likely to progress to invasive or metastatic disease. Stein et al have performed an exhaustive review of a variety of biological markers reported to have prognostic value. More recently, p53 and other cell cycle control genes, chorionic gonadotropin beta gene transcripts, various cell matrix and adhesion proteins and differentially expressed NACB.

\section{Role of urine markers for patient surveillance}

Many reports have established the value of urine tumor marker tests in the early detection of recurrent bladder tumors, but as yet these urine tests cannot replace routine cystoscopy and cytology in the management of bladder cancer patients. Instead, they may be used as complementary adjuncts that direct more effective utilization of clinical procedures, thus reducing the cost of patient surveillance. Patients with superficial lesions of low grade (Ta, Grade 1 and II) are at lower risk for recurrence than patients with Ta Grade III and T1 tumors, and these lower-risk patients may need less intensive follow-up .

The urine markers used in patient surveillance have on occasion been criticized for their low sensitivity in detecting disease, but in most studies they have significantly improved the detection of bladder cancer when used in conjunction with cytology and cystoscopy. Voided urine cytology has its own limitations in detecting carcinoma in situ (cis) and low-grade bladder tumors. It appears that urine markers can assist in the early detection of recurrence in patients with carcinoma in situ and low-grade superficial tumors.

\section{Conclusion}

The availability of many new markers for bladder cancer raises the possibility of improving the rate of cancer detection by combined use of selected markers, measured either simultaneously or sequentially. The objective of such panel testing should be to improve both the sensitivity and the specificity for bladder cancer detection. Prospective clinical trials are undoubtedly necessary to prove their clinical value, before such panels could be implemented in routine patient care. It should also be noted that the stability of these tumour marker antigens must be better defined in order to minimize false negative test results. Improved definition of the disease conditions which can produce false positive test results for urine based markers could lead to more effective use of these tests for cancer detection. It seems a long way before these markers replace invasive testing, but at least it can help define those group of patients who need cystoscopic surveillance while sparing the majority of patients who do not need the procedure. This will bring enormous cost saving to 
the increasing health care cost we face in the presence of dwinding health care budget allocations from other competing needs.

\section{References}

[1] Bailey MJ. Urinary markers in bladder cancer. BJUI 2003;91:772-773.

[2] Eissa S, Kassim S, El-Ahmady O. Detection of bladder tumours: role of cytology, morphology-based assays, biochemical and molecular markers. Curr Opin Obstet Gynecol 2003;15:395-403.

[3] Fritsche HA. Bladder cancer and urine tumor marker tests. Diamandis EP Fritsche HA Lilja H Chan DW Schwartz MK eds. Tumor markers: physiology, pathobiology, technology and clinical applications 2002:281-286 AACC Press Washington.

[4] Kageyama S, Isono T, Iwaki H, Wakabayashi Y, Okada Y, Kontani K, et al. Identification by proteomic analysis of calreticulin as a marker for bladder cancer and evaluation of the diagnostic accuracy of its detection in urine. Clin Chem 2004;50:857-866.

[5] Celis A, Rasmussen HH, Celis P, Basse B, Lauridsen JB, Ratz G, et al. Short-term culturing of low-grade superficial bladder transitional cell carcinomas leads to changes in the expression levels of several proteins involved in key cellular activities. Electrophoresis 1999;20:355-361.

[6] Bravaccini S, Sanchini MA, Granato AM, Gunelli R, Nanni O, Amadori D, Calistri D, Silvestrini R. Urine telomerase activity for the detection of bladder cancer in females. J Urol. 2007 Jul;178(1):57-61.

[7] Sanchini MA, Gunelli R, Nanni O, Bravaccini S, Fabbri C, Sermasi A, Bercovich E, Ravaioli A, Amadori D, Calistri D. Relevance of urine telomerase in the diagnosis of bladder cancer. JAMA. 2005 Oct 26;294(16):2052-6.

[8] Sanchini MA, Bravaccini S, Medri Urine telomerase: an important marker in the diagnosis of bladder cancer.

[9] Roberta Gunelli, ; Oriana Nanni, ; Sara Bravaccini, ; Carla Fabbri, Alice Sermasi, Eduard Bercovich, Alberto Ravaioli,; Dino Amadori,; Daniele Calistri. JAMA. 2005;294:2052-2056.

[10] Van Le TS, Myers J, Konety BR, Barder T, Getzenberg RH. Functional characterization of the bladder cancer marker, BLCA-4. J.Clin Cancer Res. $2004 ; 15 ; 10(4): 1384-1391$.

[11] Messing EM, Teot L, Korman H, Underhill E, Barker E, Stork B, Qian J, Bostwick DG. J Urol. 2005;174:1238-41.

[12] Oeda T, Manabe D. Nippon Hinyokika Gakkai Zasshi [The usefulness of urinary FDP in the diagnosis of bladder cancer: comparison with NMP22, BTA and cytology]. 2001;92(1):1-5.

[13] Tilki D, Burger M, Dalbagni G, Grossman HB, Hakenberg OW, Palou J, Reich O, Rouprêt M, Shariat SF, Zlotta AR. Urine Markers for Detection and Surveillance of Non-Muscle- Invasive Bladder Cancer. Eur Urol. 2011 Jun 12.

[14] Khadjavi A, Barbero G, Destefanis P, Mandili G, Giribaldi G, Mannu F, Pantaleo A, Ceruti C, Bosio A, Rolle L, Turrini F, Fontana D. Evidence of abnormal tyrosine phosphorylated proteins in the urine of patients with bladder cancer: the road toward a new diagnostic tool? J Urol. 2011 May;185(5):1922-1929.

[15] Sagnak L, Ersoy H, Gucuk O, Ozok U, Topaloglu H. Diagnostic Value of a Urine-Based Tumor Marker for Screening Lower Urinary Tract in Low-Risk Patients with Asymptomatic Microscopic Haematuria. Urol Int. 2011 Jun 3. 
[16] Yamada Y, Enokida H, Kojima S, Kawakami K, Chiyomaru T, Tatarano S, Yoshino H, Kawahara K, Nishiyama K, Seki N, Nakagawa M. MiR-96 and miR-183 detection in urine serve as potential tumor markers of urothelial carcinoma: correlation with stage and grade, and comparison with urinary cytology. Cancer Sci. 2011 Mar;102(3):522-529.

[17] Kehinde EO, Al-Mulla F, Kapila K, Anim JT. Comparison of the sensitivity and specificity of urine cytology, urinary nuclear matrix protein-22 and multi-target fluorescence in situ hybridization assay in the detection of bladder cancer. Scand J Urol Nephrol. 2011 Mar;45(2):113-121.

[18] Roobol MJ, Bangma CH, el Bouazzaoui S, Franken-Raab CG, Zwarthoff EC. Urol Oncol. 2010 Nov-Dec;28(6):686-90. Feasibility study of screening for bladder cancer with urinary molecular markers (the BLU-P project).

[19] Costa VL, Henrique R, Danielsen SA, Duarte-Pereira S, Eknaes M, Skotheim RI, Rodrigues A, Magalhães JS, Oliveira J, Lothe RA, Teixeira MR, Jerónimo C, Lind GE. Three epigenetic biomarkers, GDF15, TMEFF2, and VIM, accurately predict bladder cancer from DNA-based analyses of urine samples. Clin Cancer Res. 2010 Dec 1;16(23):5842-51.

[20] Margel D, Pesvner-Fischer M, Baniel J, Yossepowitch O, Cohen IR. Stress proteins and cytokines are urinary biomarkers for diagnosis and staging of bladder cancer. Eur Urol. 2011 Jan;59(1):113-119.

[21] Szarvas T, Singer BB, Becker M, Vom Dorp F, Jäger T, Szendroi A, Riesz P, Romics I, Rübben H, Ergün S. Urinary matrix metalloproteinase-7 level is associated with the presence of metastasis in bladder cancer. BJU Int. 2011 Apr;107(7):1069-73.

[22] Lotan Y, Shariat SF, Schmitz-Dräger BJ, Sanchez-Carbayo M, Jankevicius F, Racioppi M, Minner SJ, Stöhr B, Bassi PF, Grossman HB. Considerations on implementing diagnostic markers into clinical decision making in bladder cancer. Urol Oncol. 2010 Jul-Aug;28(4):441-8.

[23] Yutkin V, Nisman B, Pode D. Can urinary biomarkers replace cystoscopic examination in bladder cancer surveillance? Expert Rev Anticancer Ther. 2010 Jun;10(6):787-90.

[24] Lai Y, Ye J, Chen J, Zhang L, Wasi L, He Z, Zhou L, Li H, Yan Q, Gui Y, Cai Z, Wang X, Guan Z. UPK3A: a promising novel urinary marker for the detection of bladder cancer. Urology. 2010 Aug;76(2):514.6-11.

[25] Horstmann M, Bontrup H, Hennenlotter J, Taeger D, Weber A, Pesch B, Feil G, Patschan O, Johnen G, Stenzl A, Brüning T. Clinical experience with survivin as a biomarker for urothelial bladder cancer.World J Urol. 2010 Jun;28(3):399-404.

[26] Tsui KH, Tang P, Lin CY, Chang PL, Chang CH, Yung BY. Bikunin loss in urine as useful marker for bladder carcinoma. J Urol. 2010 Jan;183(1):339-44.

[27] Mengual L, Burset M, Ars E, Lozano JJ, Villavicencio H, Ribal MJ, Alcaraz A. DNA microarray expression profiling of bladder cancer allows identification of noninvasive diagnostic markers. J Urol. 2009 Aug;182(2):741-748.

[28] Lotan Y, Elias K, Svatek RS, Bagrodia A, Nuss G, Moran B, Sagalowsky AI. Bladder cancer screening in a high risk asymptomatic population using a point of care urine based protein tumour marker. J Urol. 2009 Jul;182(1):52-7; discussion 58.

[29] Svatek RS, Karam J, Karakiewicz PI, Gallina A, Casella R, Roehrborn CG, Shariat SF. Role of urinary cathepsin $\mathrm{B}$ and $\mathrm{L}$ in the detection of bladder urothelial cell carcinoma. J Urol. 2008 Feb;179(2):478-84; discussion 484. 
[30] Cai T, Mazzoli S, Meacci F, Tinacci G, Nesi G, Zini E, Bartoletti R. Interleukin-6/10 ratio as a prognostic marker of recurrence in patients with intermediate risk urothelial bladder carcinoma. J Urol. 2007 Nov;178(5):1906-11;discussion 1911-2.

[31] Yossepowitch O, Herr HW, Donat SM. Use of urinary biomarkers for bladder cancer surveillance: patient perspectives. J Urol. 2007 Apr;177(4):1277-82; discussion 1282.

[32] Shariat SF, Marberger MJ, Lotan Y, Sanchez-Carbayo M, Zippe C, Lüdecke G, Boman H, Sawczuk I, Friedrich MG, Casella R, Mian C, Eissa S, Akaza H, Serretta V, Huland H, Hedelin H, Raina R, Miyanaga N, Sagalowsky AI, Roehrborn CG, Karakiewicz PI. Variability in the performance of nuclear matrix protein 22 for the detection of bladder cancer. J Urol. 2006 Sep;176(3):919-26; discussion 926.

[33] Margel D, Pesvner-Fischer M, Baniel J, Yossepowitch O, Cohen IR. Stress proteins and cytokines are urinary biomarkers for diagnosis and staging of bladder cancer. Eur Urol. 2011 Jan;59(1):113-9.

[34] Renard I, Joniau S, van Cleynenbreugel B, Collette C, Naômé C, Vlassenbroeck I, Nicolas H, de Leval J, Straub J, Van Criekinge W, Hamida W, Hellel M, Thomas A, de Leval L, Bierau K, Waltregny D. Identification and validation of the methylated TWIST1 and NID2 genes through real-time methylation-specific polymerase chain reaction assays for the noninvasive detection of primary bladder cancer in urine samples. Eur Urol. 2010 Jul;58(1):96-104.

[35] Tilki D, Singer BB, Shariat SF, Behrend A, Fernando M, Irmak S, Buchner A, Hooper AT, Stief CG, Reich O, Ergün S. CEACAM1: a novel urinary marker for bladder cancer detection. Eur Urol. 2010 Apr;57(4):648-654.

[36] van Rhijn BW. Considerations on the use of urine markers for bladder cancer. Eur Urol. 2008 May;53(5):880-881.

[37] Vrooman OP, Witjes JA. Urinary markers in bladder cancer. Eur Urol. 2008 May;53(5):909-916.

[38] Fernandez-Gomez J, Rodríguez-Martínez JJ, Barmadah SE, García Rodríguez J, Allende DM, Jalon A, Gonzalez R, Alvarez-Múgica M. Urinary CYFRA 21.1 is not a useful marker for the detection of recurrences in the follow-up of superficial bladder cancer. Eur Urol. 2007 May;51(5):1267-74.

[39] Golshani R, Hautmann SH, Estrella V, Cohen BL, Kyle CC, Manoharan M, Jorda M, Soloway MS, Lokeshwar VB. HAS1 expression in bladder cancer and its relation to urinary HA test. Int J Cancer. 2007 Apr 15;120(8):1712-20.

[40] Grossman HB, Blute ML, Dinney CP, Jones JS, Liou LS, Reuter VE, Soloway MS. The use of urine-based biomarkers in bladder cancer. Urology. 2006 Mar;67(3 Suppl 1):62-4.

[41] Simon MA, Lokeshwar VB, Soloway MS. Current bladder cancer tests: unnecessary or beneficial? Crit Rev Oncol Hematol. 2003 Aug;47(2):91-107. 


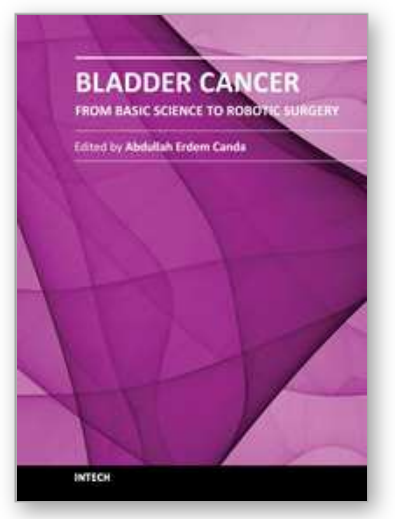

\author{
Bladder Cancer - From Basic Science to Robotic Surgery \\ Edited by Dr. Abdullah Canda
}

ISBN 978-953-307-839-7

Hard cover, 460 pages

Publisher InTech

Published online 01, February, 2012

Published in print edition February, 2012

This book is an invaluable source of knowledge on bladder cancer biology, epidemiology, biomarkers, prognostic factors, and clinical presentation and diagnosis. It is also rich with plenty of up-to-date information, in a well-organized and easy to use format, focusing on the treatment of bladder cancer including surgery, chemotherapy, radiation therapy, immunotherapy, and vaccine therapy. These chapters, written by the experts in their fields, include many interesting, demonstrative and colorful pictures, figures, illustrations and tables. Due to its practicality, this book is recommended reading to anyone interested in bladder cancer.

\title{
How to reference
}

In order to correctly reference this scholarly work, feel free to copy and paste the following:

Daben Dawam (2012). Biomarkers of Bladder Cancer in Urine: Evaluation of Diagnostic and Prognostic Significance of Current and Potential Markers, Bladder Cancer - From Basic Science to Robotic Surgery, Dr. Abdullah Canda (Ed.), ISBN: 978-953-307-839-7, InTech, Available from:

http://www.intechopen.com/books/bladder-cancer-from-basic-science-to-robotic-surgery/biomarkers-ofbladder-cancer-in-urine-evaluation-of-diagnostic-and-prognostic-significance-of-curren

\section{INTECH}

open science | open minds

\section{InTech Europe}

University Campus STeP Ri

Slavka Krautzeka 83/A

51000 Rijeka, Croatia

Phone: +385 (51) 770447

Fax: +385 (51) 686166

www.intechopen.com

\section{InTech China}

Unit 405, Office Block, Hotel Equatorial Shanghai

No.65, Yan An Road (West), Shanghai, 200040, China

中国上海市延安西路65号上海国际贵都大饭店办公楼405单元

Phone: +86-21-62489820

Fax: +86-21-62489821 
(C) 2012 The Author(s). Licensee IntechOpen. This is an open access article distributed under the terms of the Creative Commons Attribution 3.0 License, which permits unrestricted use, distribution, and reproduction in any medium, provided the original work is properly cited. 